\title{
Improving data reporting to NHMIS from the private sector in Nigeria: Lessons from a DHIS2 pilot with community pharmacists and patent and proprietary medicine vendors
}

Population Council

Follow this and additional works at: https://knowledgecommons.popcouncil.org/departments_sbsr-rh

Part of the Health Services Research Commons

How does access to this work benefit you? Let us know!

\section{Recommended Citation}

"Improving data reporting to NHMIS from the private sector in Nigeria: Lessons from a DHIS2 pilot with community pharmacists and patent and proprietary medicine vendors," IntegratE Project brief. Abuja:

Population Council, 2020. 


\section{BACKGROUND}

Governments and non-governmental organizations all over the world are investing resources and efforts to improve the quality and reporting of health services data. With reliable data, Ministries of Health can inform strategic planning and monitor progress towards health goals. In Nigeria, the Federal Ministry of Health (FMoH) adopted District Health Information System (DHIS2) as its Health Information Management Systems (HMIS) platform in 2004. The DHIS2 allows public health facilities to enter service statistics monthly using an electronic platform. These data then become available for analysis and use by decision-makers. While there are a few private facilities that use DHIS2 routinely, the National HMIS does not currently capture data from a large portion of private sources of care, particularly from Community Pharmacies (CP) and Patent and Proprietary Medicine Vendors (PPMVs).

\section{THE INTEGRATE PROJECT}

The IntegratE Project is a 4-year initiative (2017-2021) funded by the Bill \& Melinda Gates Foundation and MSD for Mothers ${ }^{1}$ that seeks to increase access to contraceptive methods by involving the private sector (CPs and PPMVs) in family planning (FP) service delivery in Lagos and Kaduna States. IntegratE is implemented by a consortium of partners, Marie Stopes International, Planned Parenthood Federation of Nigeria, Population Council, PharmAccess, and led by the Society for Family Health. IntegratE seeks to establish a regulatory system with the Pharmacists Council of Nigeria (PCN) to ensure that CPs and PPMVs provide quality FP services, comply with FP regulations and report service statistics to the Health Information Management System (HMIS). To achieve this, the IntegratE Project in collaboration with $\mathrm{PCN}$ and the $\mathrm{FMoH}$, is implementing three main activities: (1) a pilot 3-tiered accreditation system for

1 This programme is co-funded by, developed and is being implemented in collaboration with MSD for Mothers, MSD's $\$ 500$ million initiative to help create a world where no woman dies giving life. MSD for Mothers is an initiative of Merck \& Co., Inc., Kenilworth, NJ, U.S.A
PPMVs based on their healthcare qualifications; (2) pilot a hub-and-spoke supervisory model to ensure standard drug stocking practices; and (3) building the capacity of CPs and PPMVs to provide a wider range of FP services and report data to the HMIS. This brief focuses on the IntegratE Project's work towards building the capacity of CPs and PPMVs to record and report FP service statistics through a pilot DHIS2 mobile platform. Data for this brief were collected from the DHIS, Data Quality Assurance (DQA) reports and from short interviews with CPs and PPMVs during data review meetings and phone conversations. Consent was obtained prior to the interviews, and CPs and PPMVs were aware that their quotes would be used, in verbatim, in a report without their names being mentioned. Additional information on IntegratE Project can be found on www.integrateeproject.org.ng

\section{DHIS2 PILOT WITH CPS AND PPMVS}

The IntegratE Project is collaborating with the $\mathrm{FMoH}$ to pilot a DHIS2 mobile platform with CPs and PPMVs in Kaduna and Lagos states. As part of the pilot program, the IntegratE Project is building the capacity of CPs and PPMVs to accurately document and report FP services that they provide. By the end of the project, IntegratE aims to:

1. Train CPs and PPMVs to accurately document the FP services provided;

2. Facilitate CPs and PPMVs' timely data reporting that will contribute to contraceptive uptake estimates in Lagos and Kaduna;

3. Increase private-sector participation in data reporting within the national health sector using DHIS2 mobile platform

4. Support ownership and accountability of the pilot DHIS2 program by state-level stakeholders with support from the FMoH. 


\section{THE INTEGRATE PROGRESS TO DATE}

In 2018, the FMoH through the Directorate of Planning and Research, approved the NHMIS to include data from CPs and PPMVs starting with a pilot data repository. To support the implementation of this approval, the IntegratE project conducted the following activities.

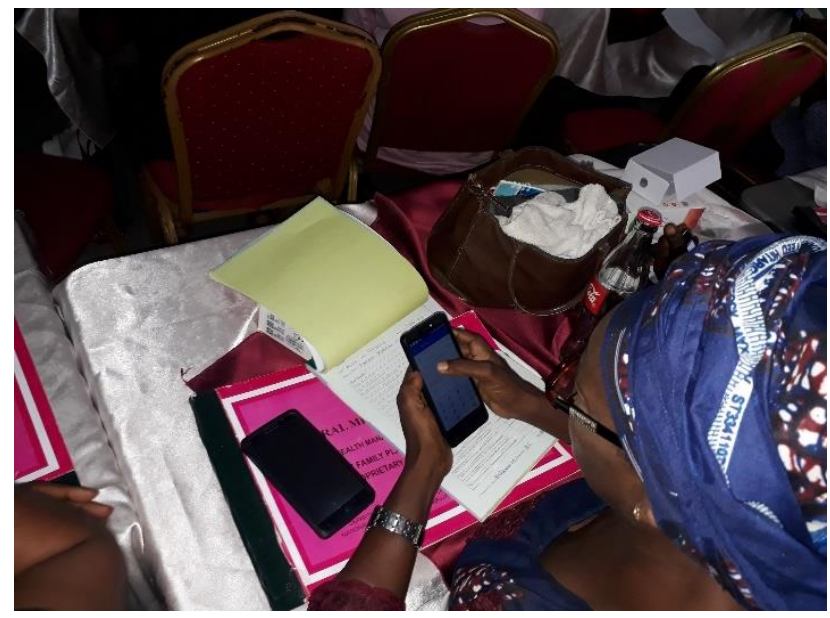

Figure1: Data Entry into DHIS2 Mobile phone

\section{Train CPs and PPMVs to report FP service} statistics using the NHMIS tools

Between July 2018 and June 2019, 894 CPs and PPMVs were trained to report FP service statistics using the NHMIS registers. The training covered: (a) the importance of proper documentation and reporting of service statistics; (b) how to complete the NHMIS registers; and (c) how to complete the NHMIS Monthly Summary sheets. CPs and PPMVs were trained to report FP service statistics in a timely fashion to Local Government Areas (LGAs) and to the IntegratE Project's Monitoring and Evaluation (M\&E) team. Between July 2018 and March 2019, trained CPs and PPMVs successfully reported FP service statistics using the NHMIS tools.

AS OF JANUARY 2020, 735 CPS AND PPMVS HAVE BEEN TRAINED ON DHIS2, WHICH IS 82\% OF THE TOTAL PROVIDERS ENGAGED BY THE INTEGRATE PROJECT $(\mathrm{N}=894)$.

\section{Train CPs and PPMVs to report FP service statistics using the mobile DHIS2}

In April 2019, the pilot DHIS2 mobile platform was developed by a FMOH DHIS Consultant to improve CPs and PPMVs monthly reporting of FP service statistics. CPs and PPMVs were trained on how to electronically report FP service statistics into the DHIS2 mobile tool. After the training, all $735 \mathrm{CPs}$ and PPMVs were provided with mobile phones equipped with the DHIS2 platform.

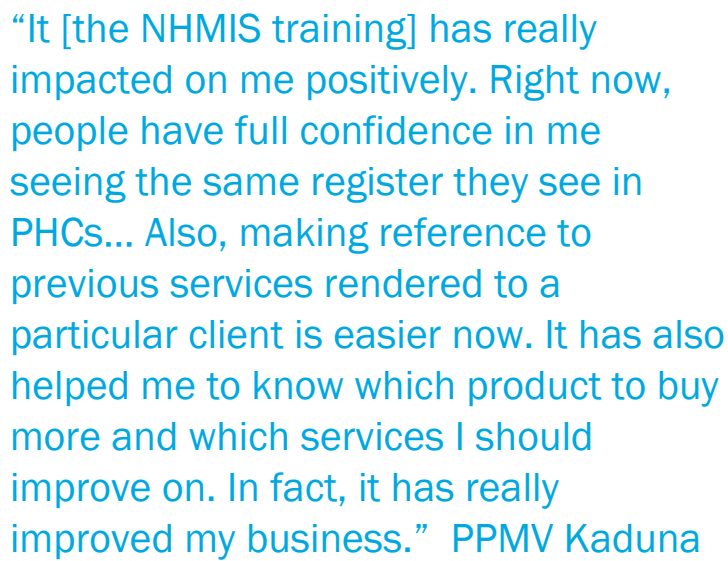

At the end of each reporting month, CPs and PPMVs completed summary FP reports, which were reviewed by the M\&E team before being uploaded to the DHIS2

In general, the majority of the IntegratE CPs and PPMVs found the mobile DHIS2 easy to use, less cumbersome than the paper-based process, and were excited to use it. They also found the DHIS2 reliable and safe for data storage. Few observed that the platform can be unresponsive if the internet network is poor. When asked about the value of the DHIS2, common responses included: the ease of data reporting and documentation; timeliness in reporting; improved reporting and recordkeeping capacity; and better stock control and inventory process.

\section{"DHIS has been very useful to me and it is stress-free because I don't have to take the report the health family for submission" PPMV Lagos}

Ensuring data quality: data verification, data quality assurance and mentorship The M\&E team conducted quarterly review meetings and a DQA process to ensure that reported FP data were of high quality. During the quarterly review meetings, FP data were reviewed for completeness and accuracy. FP data reported in the DHIS2 were also matched to the paper-based FP reporting forms. The DQA process included periodic data verification and validation exercises with randomly selected providers. They were conducted in collaboration with the State $\mathrm{MoH}$ and Primary Health Care Board officials to encourage joint supportive supervision and sustainability. 
AS OF JANUARY 2020, THE INTEGRATE M\&E TEAM HAS CONDUCTED DQA VISITS TO 104 RANDOMLY SELECTED CPS AND PPMVS AND VERIFIED DATA FROM 636 CPS AND PPMVS

The DQAs focused on 1) availability and accessibility of FP registers, FP client folders, bin cards, and monthly summary forms; 2) data consistency (consistent data from one form to a monthly summary sheet); and 3) data validity (verification of reported data versus actual data in every shop). At the end of each visit, DQA findings were discussed with the CP or PPMV, who were also given a copy of the DQA checklist summary sheet and an action plan to improve data quality moving forward. The data verification exercises and DQA visits have been very valuable to CPs and PPMVs as suggested from these quotes:

In addition to the data verification exercises and DQA, the IntegratE Project provided continual support and mentorship to CPs and PPMVs through WhatsApp, peerto-peer mentorship and Supportive Supervision to ensure CPs and PPMVs could report quality FP data (Box 1).

\section{Box 1: IntegratE data reporting support}

WhatsApp allowed the project to provide continuous remote support for record keeping and data reporting. Providers could ask questions and the M\&E team could address issues in real time.

The M\&E team identified champion CPs and PPMVs who demonstrated strong understanding of and capacity for record and reporting quality data. Starting in April 2019, these champions served as peer mentors for other providers in their area and supported those who could not accurately complete their monthly reports on time.

From July 2018-June 2019, the M\&E team completed supportive supervision visits to CPs and PPMVs to provide on-site mentorship for proper record keeping accurate completion of NHMIS registers.
Figure 2 : Proportion of CPs and PPMVs who reported into the DHIS2 mobile phones

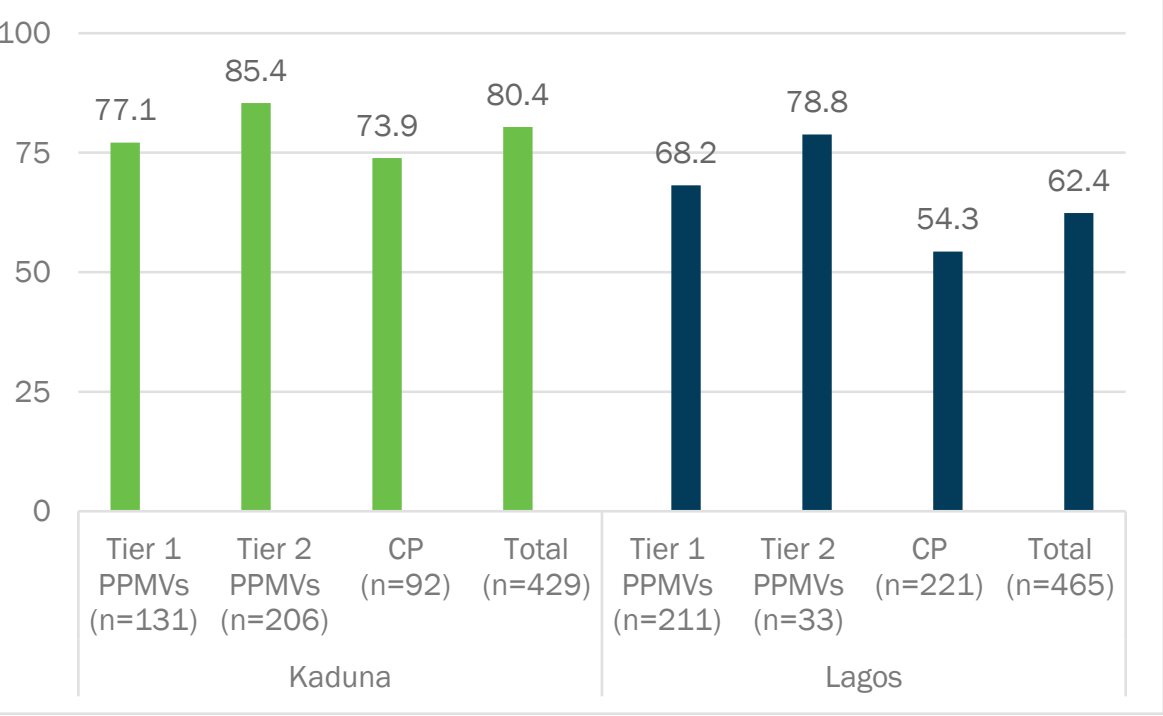

\section{REPORTING RATES}

Figure 2 shows the reporting rates of IntegratE CPs and PPMVs. Average CP and PPMV monthly reporting rates using the mobile DHIS2 between April - September 2019 ranged between 62 and 80 percent, with about 54 percent of all CPs and PPMVs reporting on time (data not shown). Reporting in both states are similar though slightly better in Kaduna state. Figure 3 shows the proportion of clients receiving services from CPs and PPMVs, taken from data reported by the IntegratE providers. Between October and December of 2018, 423 trained CPs and PPMVs provided 4,374 FP services to new and existing clients. The number of services offered increased over time, which correlates to the increased number of trained CPs and PPMVs. Across all quarters reported, a higher proportion of the services offered were to women aged 25-49 years old.

\footnotetext{
"It is very important. When we see where we are wrong and correcting it with us is good" PPMV Tier 2 Alimosho

During data visit, I was corrected in certain areas of documentation. These lessons have guided me in documentation of service provision" ...PMS Kaduna South
} 
Figure 3: Proportion of clients disaggregated by age group served by CPs and PPMVs

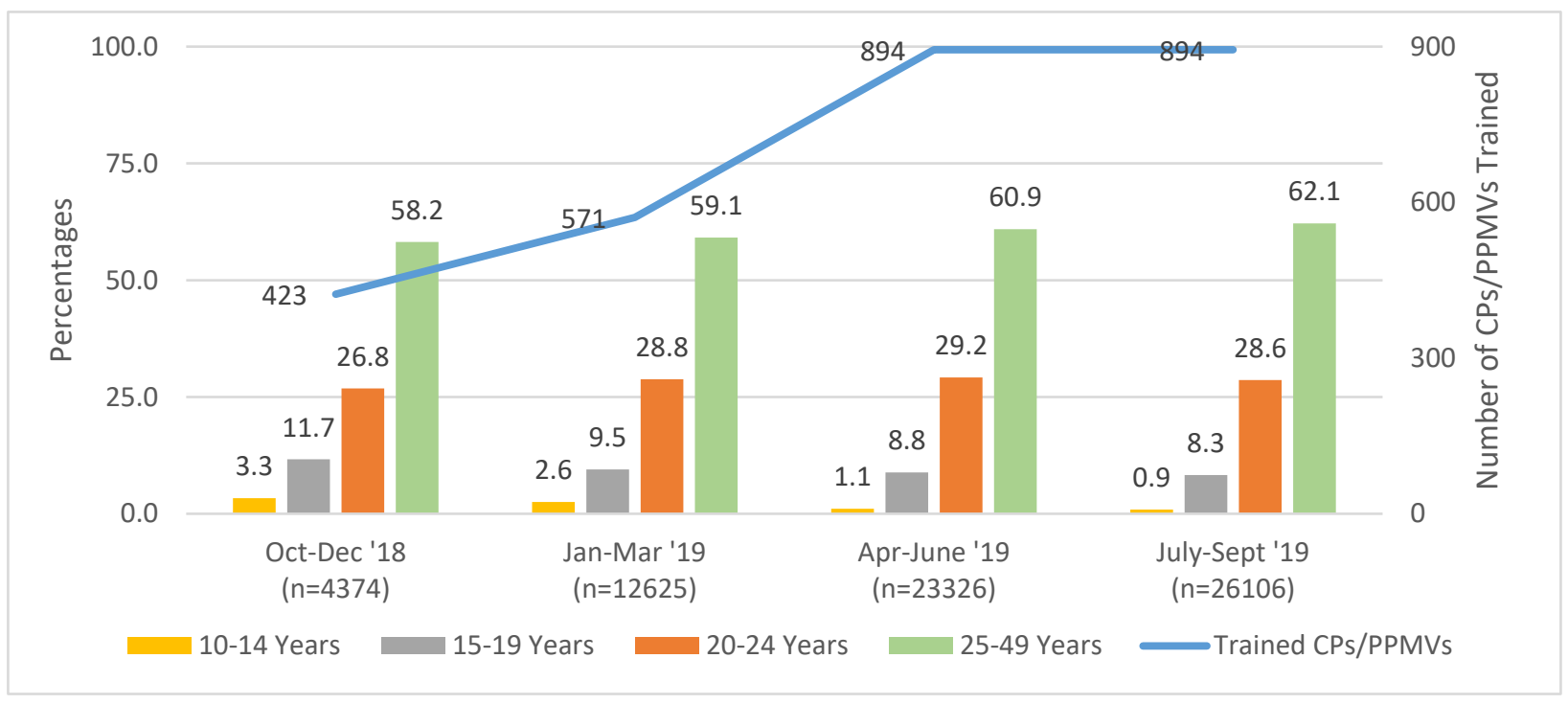

\section{CHALLENGES}

For many CPs and PPMVs, the DHIS2 is a very useful and easy to use, but connectivity issues and the number of forms to complete before monthly DHIS2 submissions were noted as challenges.

"I like it. In less than 3 minutes, I am done with my report. The only issue is all these forms that we still have to do before filling it" ...CP Ikorodu

\section{"Network is the problem I have. I will have sent the data and they will keep calling that they did not see my report" ... Tier 1 PPMV Epe}

In October 2019, the reporting format changed to reflect the revised NHMIS tools that required CPs and PPMVs to adapt to the revised tool. CPs and PPMVs are still in the process of familiarizing themselves with these changes and IntegratE is helping them navigate through this. As CPs and PPMVs began entering data using DHIS2, there were a few issues related to shops or pharmacies having duplicate or similar names. This led to data being reported incorrectly in the database for a given period.

\section{KEY LESSONS}

- The mobile DHIS2 is a scalable platform that would allow the NHMIS to include data from the private sector, specifically from CPs and PPMVs.

- A system for verifying data reported by CPs and PPMVs (DQA) is needed to ensure accurate and reliable data and to provide CPs and PPMVs with feedback to improve the quality of data reporting

- Mentoring and support is essential to improving mobile data reporting from CPs and PPMVs.

- Unique identifiers for each CP and PPMV in the DHIS2 tool are critical to avoid misreporting among shops or pharmacies with similar names.

- Data review meetings with PCN, primary health care coordinators and State $\mathrm{MoH}$ help to identify peer mentors who assist other CPs and PPMVs in the documentation and reporting

- Using the online mobile DHIS platform for data collection into NHMIS is feasible approach as many CPs and PPMVs already use mobile phones to send and receive messages and are therefore proficient in using mobile phones. 